\title{
COSMIC REIONIZATION AND EARLY STAR-FORMING GALAXIES: A JOINT ANALYSIS OF NEW CONSTRAINTS FROM PLANCK AND THE HUBBLE SPACE TELESCOPE
}

\author{
Brant E. Robertson ${ }^{1}$, Richard S. Ellis ${ }^{2}$, Steven R. Furlanetto ${ }^{3}$, and James S. Dunlop ${ }^{4}$ \\ ${ }^{1}$ Department of Astronomy, University of Arizona, Tucson, AZ 85721, USA; brant@email.arizona.edu
${ }^{2}$ Cahill Center for Astronomy and Astrophysics, California Institute of Technology, MS 249-17, Pasadena, CA 91125, USA \\ ${ }^{3}$ Department of Physics \& Astronomy, University of California, Los Angeles, Los Angeles, CA 90095, USA \\ ${ }^{4}$ Institute for Astronomy, University of Edinburgh, Edinburgh EH9 3HJ, UK \\ Received 2015 February 6; accepted 2015 March 2; published 2015 April 1
}

\begin{abstract}
We discuss new constraints on the epoch of cosmic reionization and test the assumption that most of the ionizing photons responsible arose from high-redshift star-forming galaxies. Good progress has been made in charting the end of reionization through spectroscopic studies of $z \simeq 6-8$ QSOs, gamma-ray bursts, and galaxies expected to host Ly $\alpha$ emission. However, the most stringent constraints on its duration have come from the integrated optical depth, $\tau$, of Thomson scattering to the cosmic microwave background. Using the latest data on the abundance and luminosity distribution of distant galaxies from Hubble Space Telescope imaging, we simultaneously match the reduced value $\tau=0.066 \pm 0.012$ recently reported by the Planck collaboration and the evolving neutrality of the intergalactic medium with a reionization history within $6 \lesssim z \lesssim 10$, thereby reducing the requirement for a significant population of very high redshift $(z \gg 10)$ galaxies. Our analysis strengthens the conclusion that starforming galaxies dominated the reionization process and has important implications for upcoming $21 \mathrm{~cm}$ experiments and searches for early galaxies with the James Webb Space Telescope.
\end{abstract}

Key words: galaxies: high-redshift

\section{INTRODUCTION}

Cosmic reionization represents an important era for assembling a coherent picture of the evolution of the universe, and ambitious observational facilities are being constructed to explore the most important redshift range $7<z<20$. Through the Gunn-Peterson effect in high-redshift QSOs and gamma-ray bursts (GRBs; e.g., Fan et al. 2006; Bolton et al. 2011; Chornock et al. 2013; McGreer et al. 2015) and the declining visibility of $\operatorname{Ly} \alpha$ emission in high-redshift galaxies (Stark et al. 2010; Pentericci et al. 2011, 2014; Schenker et al. 2012, 2014; Treu et al. 2013; Tilvi et al. 2014), observations indicate that reionization ended by redshift $z \simeq 6$. However, the onset and duration of the reionization process remain less certain. The most convincing constraint is provided by the integrated optical depth, $\tau$, of Thomson scattering to the cosmic microwave background (CMB). The WMAP delivered a value of $\tau=0.088 \pm 0.014$, which, in the simplest model, corresponds to "instantaneous" reionization at $z_{\text {reion }} \simeq 10.5 \pm 1.1$ (Hinshaw et al. 2013). As a result, the $W M A P$ result has been widely interpreted as implying that reionization began at $z \simeq 15$ or even earlier (Bromm \& Yoshida 2011; Dunlop 2013).

Important information on the duration of reionization can now be determined from the star formation rate (SFR) history (Madau \& Dickinson 2014, hereafter MD 14) since early starforming galaxies most likely supply the ionizing photons (Robertson et al. 2010, 2013). This conclusion followed the first measures of their abundance over $8<z<10$ from Hubble Space Telescope (HST) Ultra Deep Field (UDF) observations (Beckwith et al. 2006; Illingworth et al. 2013; Koekemoer et al. 2013). With plausible assumptions, $6<z<8$ star-forming galaxies can keep the universe substantially ionized (Robertson et al. 2013; Finkelstein et al. 2014).
However, to match the WMAP value of $\tau$, Robertson et al. (2013) also required a significant population of star-forming galaxies beyond a redshift $z \simeq 10$. As a direct census of $z>10$ galaxies is not currently possible, studies have since focused on the rate of decline in abundance over $8<z<10$ with mixed conclusions (cf. Oesch et al. 2012, 2013; McLeod et al. 2014; Ishigaki et al. 2015). The requirement for a significant contribution of ionizing photons from $z>10$ galaxies remains an important uncertainty whose resolution is perceived as a major goal for the James Webb Space Telescope (JWST).

The Planck Collaboration et al. (2015) has recently reported a significantly lower value of the optical depth, $\tau=0.066 \pm 0.012$, consistent with a reduced redshift of instantaneous reionization, $z_{\text {reion }}=8.8_{-1.1}^{+1.2}$. Here we determine the extent to which the Planck result reduces the need for significant star formation in the uncharted epoch at $z>10$. To demonstrate this, we calculate the contribution of $6<z<10$ star-forming galaxies to the integrated value of $\tau$, using the latest $H S T$ data. We then examine the residual contribution of ionizing photons required from sources beyond $z \simeq 10$ to match the new value of $\tau$ from Planck, phrasing these constraints in terms of the likely abundance of $z>10$ galaxies that JWST would see in a typical deep exposure.

Throughout, we use the AB magnitude system (Oke 1974), errors represent $1 \sigma$ uncertainties, and all cosmological calculations assume flatness and the most recent Planck cosmological parameters $\left(h=0.6774, \Omega_{m}=0.309, \Omega_{b} h^{2}=0.02230\right.$, $Y_{p}=0.2453$; Planck Collaboration et al. 2015).

\section{CONTRIBUTION OF $z<10$ GALAXIES TO LATE REIONIZATION}

\subsection{Cosmic Star Formation History}

If Lyman continuum photons from star-forming galaxies dominate the reionization process, an accounting of the 
evolving SFR density will provide a measure of the timedependent cosmic ionization rate

$$
\dot{n}_{\text {ion }}=f_{\text {esc }} \xi_{\text {ion }} \rho_{\mathrm{SFR}},
$$

where $f_{\text {esc }}$ is the fraction of photons produced by stellar populations that escape to ionize the intergalactic medium (IGM), $\xi_{\text {ion }}$ is the number of Lyman continuum photons per second produced per unit SFR for a typical stellar population, and $\rho_{\mathrm{SFR}}$ is the cosmic SFR density. Following Robertson et al. (2013), we adopt a fiducial escape fraction of $f_{\text {esc }}=0.2$ and, motivated by the rest-frame UV spectral energy distributions of $z \sim 7-8$ galaxies (Dunlop et al. 2013), a fiducial Lyman continuum photon production efficiency of $\log _{10} \xi_{\text {ion }}=53.14\left(\right.$ Lyc photons s $\left.{ }^{-1} M_{\odot}^{-1} \mathrm{yr}\right)$. Somewhat larger values of $\xi_{\text {ion }}$ may also be acceptable (e.g., Topping \& Shull 2015).

The observed infrared and rest-frame UV luminosity functions (LFs) provide a means to estimate $\rho_{\text {SFR }}$. We use the recent compilation of IR and UV LFs provided in Table 1 of MD 14 and references therein to compute luminosity densities $\rho_{L}$ to a minimum luminosity of $L_{\min }=0.001 L_{\star}$, where $L_{\star}(z)$ is the characteristic luminosity of each relevant LF parameterization (e.g., Schechter or broken power-law models). ${ }^{5}$ We supplement the MD 14 compilation by including $\rho_{\mathrm{SFR}}$ values computed from the LF determinations at $z \sim 8$ by Schenker et al. (2013), at $z \sim 7-8$ by McLure et al. (2013), and estimates at $z \sim 10$ by Oesch et al. (2014) and Bouwens et al. (2014). We include new HST Frontier Fields LF constraints at $z \sim 7$ by Atek et al. (2014) and at $z \sim 9$ by McLeod et al. (2014), incorporating cosmic variance estimates from Robertson et al. (2014). We also updated the MD 14 estimates derived from the Bouwens et al. (2012) LFs at $z \sim 3$ - 8 with newer measurements by Bouwens et al. (2014). All data were converted to the adopted Planck cosmology.

We adopted the conversion $\rho_{\mathrm{SFR}}=\kappa \rho_{L}$ supplied by MD 14 for IR and UV luminosity densities, i.e., $\kappa_{\mathrm{IR}}=1.73 \times 10^{-10}$ $M_{\odot} \mathrm{yr}^{-1} L_{\odot}^{-1}$ and $\kappa_{\mathrm{UV}}=2.5 \times 10^{-10} M_{\odot} \mathrm{yr} L_{\odot}^{-1}$ respectively, as well as their redshift-dependent dust corrections and a Salpeter initial mass function. Uncertainties on $\rho_{\mathrm{SFR}}$ are computed using faint-end slope uncertainties, where available; otherwise, we increased the uncertainties reported by MD 14 by the ratio of the luminosity densities integrated to $L=0.03 L_{\star}$ and $L=0.001 L_{\star}$. The data points in Figure 1 show the updated SFR densities and uncertainties determined from the IR (dark red) and UV (blue) LFs, each extrapolated to $L_{\text {min }}=0.001 L_{\star}$.

Since we are interested in the reionization history both up to and beyond the limit of the current observational data, we adopt the four-parameter fitting function from MD 14 to model $\rho_{\mathrm{SFR}}(z)$,

$$
\rho_{\mathrm{SFR}}(z)=a_{p} \frac{(1+z)^{b_{p}}}{1+\left[(1+z) / c_{p}\right]^{d_{p}}},
$$

and perform a maximum likelihood (ML) determination of the parameter values using Bayesian methods (i.e., Multinest;

\footnotetext{
5 We adopt this limit since it corresponds to $M_{\max } \approx-13$ at $z \sim 7$, which Robertson et al. (2013) found was required to reionize the universe by $z \sim 6$. It corresponds to $M_{\max }=M_{\star}+7.5$.
}

Feroz et al. 2009) assuming Gaussian errors. If we fit to the data and uncertainties reported by MD 14, we recover similar ML values for the parameters of Equation (2). The range of credible SFR histories can then be computed from the marginalized likelihood of $\rho_{\text {SFR }}$ by integrating over the full model parameter likelihoods.

\subsection{Thomson Optical Depth}

If photons from star-forming galaxies drive the reionization process, measures of the Thomson optical depth inferred from the CMB place additional constraints on $\rho_{\mathrm{SFR}}$. The Thomson optical depth is given by

$$
\tau(z)=c\left\langle n_{\mathrm{H}}\right\rangle \sigma_{\mathrm{T}} \int_{0}^{z} f_{\mathrm{e}} Q_{\mathrm{H}_{\mathrm{II}}}\left(z^{\prime}\right) H^{-1}\left(z^{\prime}\right)\left(1+z^{\prime}\right)^{2} d z^{\prime},
$$

where $c$ is the speed of light. The comoving hydrogen density $\left\langle n_{\mathrm{H}}\right\rangle=X_{p} \Omega_{b} \rho_{c}$ involves the hydrogen mass fraction $X_{p}$, the baryon density $\Omega_{b}$, and the critical density $\rho_{c}$. The Thomson scattering cross section is $\sigma_{T}$. The number of free electrons per hydrogen nucleus is calculated following Kuhlen \& FaucherGiguère (2012), assuming doubly ionized helium at $z \leqslant 4$.

The IGM ionized fraction $Q_{\mathrm{H}_{\mathrm{II}}}(z)$ is computed by evolving the differential equation

$$
\dot{Q}_{\mathrm{H}_{\mathrm{II}}}=\frac{\dot{n}_{\mathrm{ion}}}{\left\langle n_{\mathrm{H}}\right\rangle}-\frac{Q_{\mathrm{H}_{\mathrm{II}}}}{t_{\mathrm{rec}}}
$$

where the IGM recombination time

$$
t_{\mathrm{rec}}=\left[C_{\mathrm{H}_{\mathrm{II}}} \alpha_{\mathrm{B}}(T)\left(1+Y_{p} / 4 X_{p}\right)\left\langle n_{\mathrm{H}}\right\rangle(1+z)^{3}\right]^{-1}
$$

is calculated by evaluating the case $\mathrm{B}$ recombination coefficient $\alpha_{\mathrm{B}}$ at an IGM temperature $T=20,000 \mathrm{~K}$ and a clumping fraction $C_{\mathrm{H}_{\mathrm{II}}}=3$ (e.g., Pawlik et al. 2009; Shull et al. 2012). We incorporate the Planck Thomson optical depth constraints ( $\tau=0.066 \pm 0.012$, treated as a Gaussian) by computing the reionization history for every value of the $\rho_{\mathrm{SFR}}$ model parameters, evaluating Equation (3), and then calculating the likelihood of the model parameters given the SFR history data and the marginalized Thomson optical depth.

Figure 1 shows the ML and 68\% credibility interval (red region) on $\rho_{\mathrm{SFR}}(z)$ given the $\rho_{\mathrm{SFR}}$ constraints and the newly reported Planck Thomson optical depth. We find the parameters of Equation (2) to be $a_{p}=0.01376 \pm 0.001 M_{\odot} \mathrm{yr} \mathrm{Mpc}^{-3}, \quad b_{p}=3.26 \pm 0.21$, $c_{p}=2.59 \pm 0.14$, and $d_{p}=5.68 \pm 0.19$. Without the Thomson optical depth constraint, the values change by less than $1 \%$. These inferences can be compared with an SFR history (Figure 1, orange region) forced to match the previous WMAP measurement $(\tau=0.088 \pm 0.014)$ by upweighting the contribution of the derived $\tau$ value relative to the $\rho_{\mathrm{SFR}}$ data. The model's ML parameters $\left(a_{p}=0.01306, b_{p}=3.66, c_{p}=2.28\right.$, and $\left.d_{p}=5.29\right)$ lie well outside the range of models that reproduce jointly $\rho_{\text {SFR }}(z)$ and the Planck $\tau$. Fitting to only data at $z>3$ or only independent data points at $z>6$ changes our credibility intervals by $\sim 25 \%$.

We can now address the important question of the redshiftdependent contribution of galaxies to the Planck $\tau=0.066 \pm 0.012$ in Figure 2. The red region shows a history that is consistent with the SFR densities shown in 


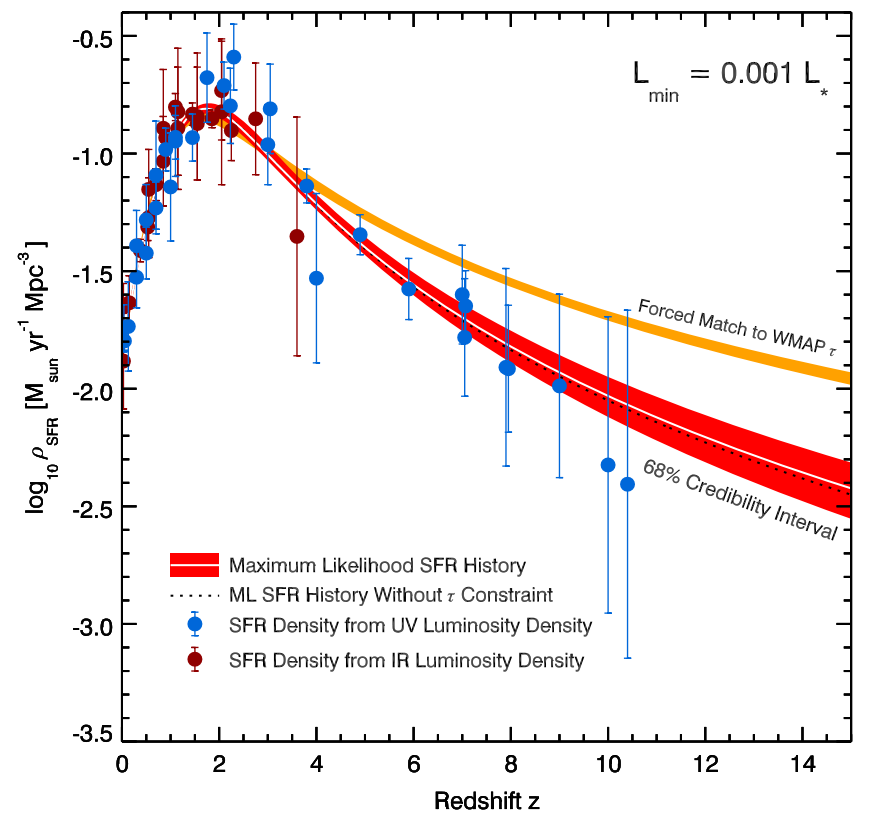

Figure 1. Star formation rate density $\rho_{\mathrm{SFR}}$ with redshift. Shown are the SFR densities from Madau \& Dickinson (2014) determined from infrared (dark red points) and ultraviolet (blue points) luminosity densities, updated for recent results and extrapolated to a minimum luminosity $L_{\min }=0.001 L_{\star}$. A parameterized model for the evolving SFR density (Equation (2)) is fit to the data under the constraint that the Thomson optical depth $\tau$ to electron scattering measured by Planck is reproduced. The maximum likelihood model (white line) and $68 \%$ credibility interval on $\rho_{\mathrm{SFR}}$ (red region) are shown. A consistent SFR density history is found even if the Planck $\tau$ constraint is ignored (dotted black line). These inferences can be compared with a model forced to reproduce the previous WMAP $\tau$ (orange region), which requires a much larger $\rho_{\mathrm{SFR}}$ at redshifts $z>5$.

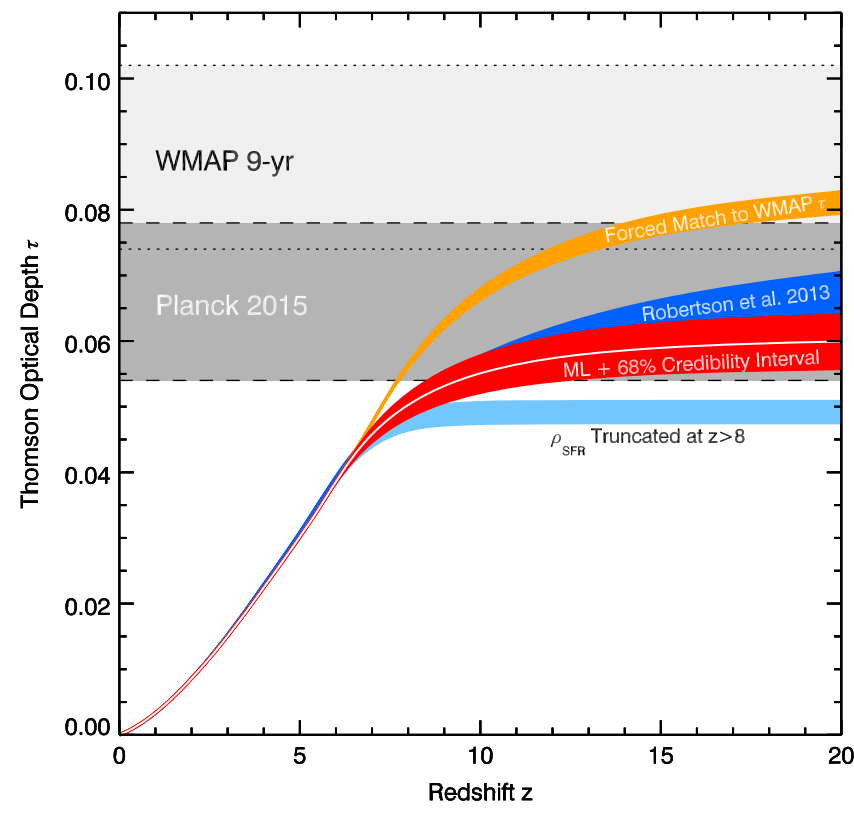

Figure 2. Thomson optical depth to electron scattering $\tau$, integrated over redshift. Shown is the Planck constraint $\tau=0.066 \pm 0.012$ (gray area), along with the marginalized $68 \%$ credibility interval (red region) computed from the SFR histories $\rho_{\mathrm{SFR}}$ shown in Figure 1. The corresponding inferences of $\tau(z)$ from Robertson et al. (2013; dark blue region), a model forced to reproduce the 9 yr WMAP $\tau$ constraints (orange region), and a model with $\rho_{\mathrm{SFR}}$ truncated at $z>8$ (light blue region) following Oesch et al. (2014) are shown for comparison.
Figure 1, given our simple assumptions for the escape fraction $f_{\text {esc }}$, early stellar populations, and the clumpiness of the IGM. Importantly, the reduction in $\tau$ by Planck (compared to $W M A P)$ largely eliminates the tension between $\rho_{\mathrm{SFR}}(z)$ and $\tau$ that was discussed by many authors, including Robertson et al. (2013). That an SFR history consistent with the $\rho_{\mathrm{SFR}}(z)$ data easily reproduces the Planck $\tau$ strengthens the conclusions of Robertson et al. (2013) that the bulk of the ionizing photons emerged from galaxies. Figure 2 shows that the observed galaxy population at $z<10$ can easily reach the $68 \%$ credibility intervals of $\tau$ with plausible assumptions about $f_{\text {esc }}$ and $L_{\min }$. As a consequence, the reduced $\tau$ eliminates the need for very high-redshift $(z \gg 10)$ star formation (see Section 3 ). We note the dust correction used in computing $\rho_{\mathrm{SFR}}$ at $z \sim 6$ permits an equivalently lower $f_{\text {esc }}$ without significant change in the derived $\tau$. We note that to reach $\tau \gtrsim 0.08$ given the $\rho_{\mathrm{SFR}}(z)$ constraints requires $f_{\text {esc }} \gtrsim 0.3$ or $C_{\mathrm{H}_{\mathrm{II}}} \lesssim 1$.

Figure 2 also shows $\tau(z)$ computed with the $9 \mathrm{yr} W M A P \tau$ marginalized likelihood as a constraint on the high-redshift SFR density (blue region; Robertson et al. 2013), which favored a relatively low $\tau \sim 0.07$. If, instead, the SFR density rapidly declines as $\rho_{\mathrm{SFR}} \propto(1+z)^{-10.9}$ beyond $z \sim 8$ as suggested by, e.g., Oesch et al. (2014), the Planck $\tau$ is not reached (light blue region). Finally, if we force the model to reproduce the best-fit WMAP $\tau$ (orange region), the increased ionization at high redshifts requires a dramatic increase in the $z>7.5$ SFR (see Figure 1) and poses difficulties in matching other data on the IGM ionization state, as we discuss next.

\subsection{Ionization History}

Similarly, we can update our understanding of the evolving ionization fraction $Q_{\mathrm{H}_{\mathrm{II}}}(z)$ computed during the integration of Equation (4). Valuable observational progress in this area made in recent years exploits the fraction of star-forming galaxies showing Ly $\alpha$ emission (e.g., Stark et al. 2010) now extended to $z \sim 7-8$ from Treu et al. (2013), Pentericci et al. (2014), and Schenker et al. (2014); the Ly $\alpha$ damping wing absorption constraints from GRB host galaxies by Chornock et al. (2013); and the number of dark pixels in Ly $\alpha$ forest observations of background quasars (McGreer et al. 2015). While most of these results require model-dependent inferences to relate observables to $Q_{\mathrm{H}_{\mathrm{II}}}$, they collectively give strong support for reionization ending rapidly near $z \simeq 6$.

Figure 3 shows these constraints, along with the inferred $68 \%$ credibility interval (red region; ML model shown in white) on the marginalized distribution of the neutral fraction $1-Q_{\mathrm{H}_{\mathrm{II}}}$ from the SFR histories shown in Figure 1 and the Planck constraints on $\tau$. Although our model did not use these observations to constrain the computed reionization history, we nonetheless find good agreement. ${ }^{6}$

Figure 3 also shows the earlier model of Robertson et al. (2013; blue region) that completes reionization at a slightly lower redshift and displays a more prolonged ionization history. This model was in some tension with the WMAP $\tau$ (Figure 2). If we force the model to reproduce the WMAP $\tau$ (orange region), reionization ends by $z \sim 7.5$, which is quite inconsistent with several observations that indicate neutral gas within IGM over the range $6 \lesssim z \lesssim 8$ (Figure 3 ).

\footnotetext{
6 The model does not fare well in comparison to Ly $\alpha$ forest measurements when $Q_{\mathrm{H}_{\text {II }}} \sim 1$ because of our simplified treatment of the ionization process (see the discussion in Robertson et al. 2013).
} 


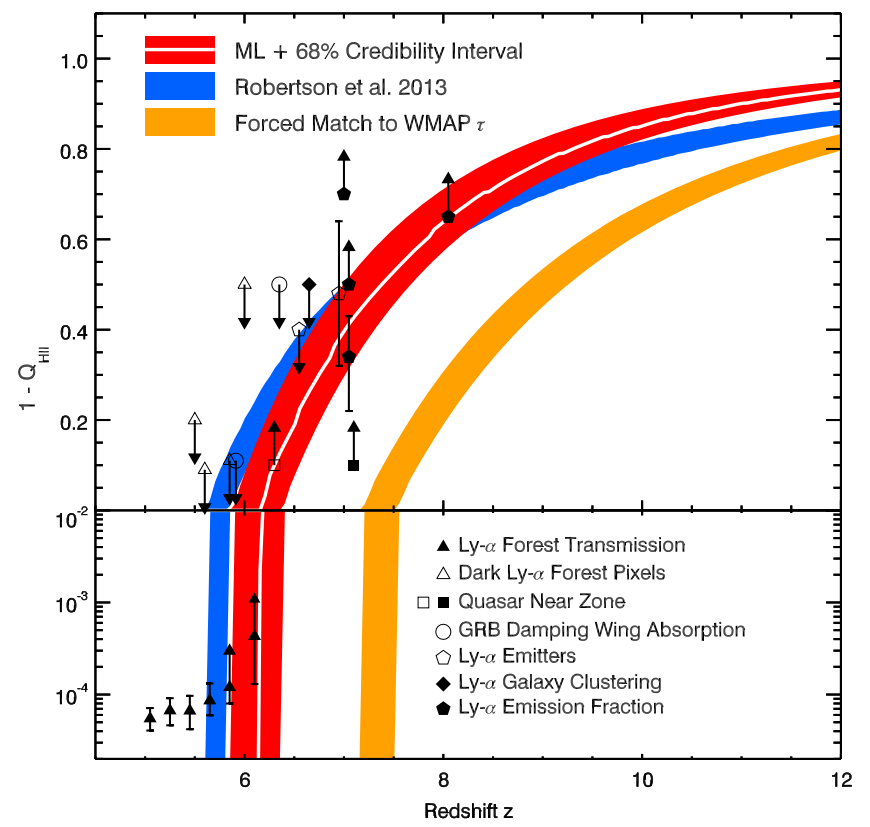

Figure 3. Measures of the neutrality $1-Q_{\mathrm{H}_{\text {II }}}$ of the intergalactic medium as a function of redshift. Shown are the observational constraints compiled by Robertson et al. (2013), updated to include recent IGM neutrality estimates from the observed fraction of $\operatorname{Ly} \alpha$ emitting galaxies (Pentericci et al. 2014; Schenker et al. 2014), constraints from the Ly $\alpha$ of GRB host galaxies (Chornock et al. 2013), and inferences from dark pixels in Ly $\alpha$ forest measurements (McGreer et al. 2015). The evolving IGM neutral fraction computed by the model is also shown (red region is the 68\% credibility interval; white line is the ML model). While these data are not used to constrain the models, they are nonetheless remarkably consistent. The bottom panel shows the IGM neutral fraction near the end of the reionization epoch, where the presented model fails to capture the complexity of the reionization process. For reference, we also show the corresponding inferences calculated from Robertson et al. (2013; blue region) and a model forced to reproduce the $W M A P \tau$ (orange region).

\section{CONSTRAINTS ON THE CONTRIBUTION OF $z>10$ GALAXIES TO EARLY REIONIZATION}

By using the parameterized model of MD 14 to fit the cosmic SFR histories and applying a simple analytical model of the reionization process, we have demonstrated that SFR histories consistent with the observed $\rho_{\mathrm{SFR}}(z)$ integrated to $L_{\min }=0.001 L_{\star}$ reproduce the observed Planck $\tau$ while simultaneously matching measures of the IGM neutral fraction at redshifts $6 \lesssim z \lesssim 8$. As Figure 1 makes apparent, the parameterized model extends the inferred SFR history to $z>10$, beyond the reach of current observations. Correspondingly, these galaxies supply a non-zero rate of ionizing photons that enable the Thomson optical depth to slowly increase beyond $z \sim 10$ (see Figure 2). We can therefore ask whether a connection exists between $\rho_{\mathrm{SFR}}(z>10)$ and the observed value of $\tau$ under the assumption that star-forming galaxies control the reionization process.

Figure 4 shows samples from the likelihood function of our model parameters given the $\rho_{\mathrm{SFR}}(z)$ and $\tau$ empirical constraints that indicate the mean SFR density $\left\langle\rho_{\mathrm{SFR}}\right\rangle$ (averaged over $10 \lesssim z \lesssim 15)$ as a function of the total Thomson optical depth $\tau$. The properties $\left\langle\rho_{\mathrm{SFR}}\right\rangle$ and $\tau$ are tightly related, such that the linear fit

$$
\left\langle\rho_{\mathrm{SFR}}\right\rangle \approx 0.344(\tau-0.06)+0.00625\left(M_{\odot} \mathrm{yr}^{-1} \mathrm{Mpc}^{-3}\right)
$$

provides a good description of their connection (dashed line). For reference, the likelihood samples shown in Figure 4 indicate the corresponding redshift of instantaneous reionization $z_{\text {reion }}$ via color coding.

Given that the SFR density is supplied by galaxies that are luminous in their rest-frame UV, we can also connect the observed $\tau$ to the abundance of star-forming galaxies at $z \gtrsim 10$. This quantity holds great interest for future studies with JWST, as the potential discovery and verification of distant galaxies beyond $z>10$ has provided a prime motivation for the observatory. The $5 \sigma$ sensitivity of JWST at $2 \mu \mathrm{m}$ in a $t=10^{4} \mathrm{~s}$ exposure is $m_{\mathrm{AB}} \approx 29.5$. $^{7}$ At $z \sim 10$, this sensitivity corresponds to a UV absolute magnitude of $M_{\mathrm{UV}} \approx-18$. Extrapolating the SFR density to $z>10$ and using the shape of the LF at $z \geqslant 9$, we estimate that $N \sim 0.5 \operatorname{arcmin}^{-2}$ galaxies at $z>10$ will be present at apparent magnitudes of $m_{\mathrm{AB}}<29.5$ at $\lambda=2 \mu \mathrm{m}$. Deep observations with JWST over $\sim 10 \mathrm{arcmin}^{2}$ may therefore find $\gtrsim 5$ candidates at $z>10$ (see also Behroozi $\&$ Silk 2015). Returning to Figure 1, we can see the impact of the reduced value of $\tau$ by comparing the Planck and WMAP curves beyond $z \simeq 10$.

\section{DISCUSSION}

The lower value of the optical depth $\tau$ of Thomson scattering reported by the Planck Collaboration et al. (2015) strengthens the likelihood that early star-forming galaxies dominated the reionization process, as our model can simultaneously match the observed SFR history (Figure 1) over $6<z<10$, the integrated value of $\tau$ (Figure 2 ), and recent constraints on the IGM neutral fraction over $z \simeq 6-8$ (Figure 3 ).

A state-of-the-art reionization analysis by Choudhury et al. (2014) used the distribution of Ly $\alpha$ equivalent widths, the IGM photoionization rate, and the mean free path of ionizing photons to also conclude that reionization likely completed at $z \sim 6$, with a corresponding $\tau \approx 0.07$ (see also Robertson et al. 2013). With Planck now favoring $\tau \approx 0.066$ and informed by a full accounting of available constraints on the SFR history, we have reached similar conclusions using different empirical inputs.

Our modeling makes some simplifying assumptions, adopting a constant escape fraction $f_{\mathrm{esc}}=0.2$, IGM clumping factor $C \approx 3$, and Lyman continuum production efficiency for early stellar populations. In Robertson et al. (2013), we examined these assumptions carefully and tested more complex models, e.g., with the evolving escape fraction required to match the IGM photoionization rates at $z<6$ (e.g., Becker \& Bolton 2013). These assumptions influence the computation of $\tau$ and $Q_{\mathrm{H}_{\mathrm{II}}}$ but do not affect the inferred SFR history in Figure 1. Our conclusion that $z \lesssim 10$ galaxies can account for the Planck $\tau$ relies on extrapolating LFs below observed limits and a higher escape fraction than at a lower redshift. If galaxies are less efficient ionizers, more $z>10$ star formation would be permitted. However, Robertson et al. (2013) already demonstrated such an ionizing efficiency is required to maintain a highly ionized IGM at $z \sim 7$ (Figure 3).

The "excess" value of $\tau$ above that provided by galaxies at $z<10$ measures $\rho_{\mathrm{SFR}}$ at $z>10$. Equation (6) and the Planck $1 \sigma$ upper limit on $\tau$ provide an upper limit of $\rho_{\mathrm{SFR}}(z>10) \lesssim 0.013 M_{\odot} \mathrm{yr}^{-1} \mathrm{Mpc}^{-3}$. This provides the first

\footnotetext{
See http://www.stsci.edu/jwst/instruments/nircam/sensitivity/table.
} 


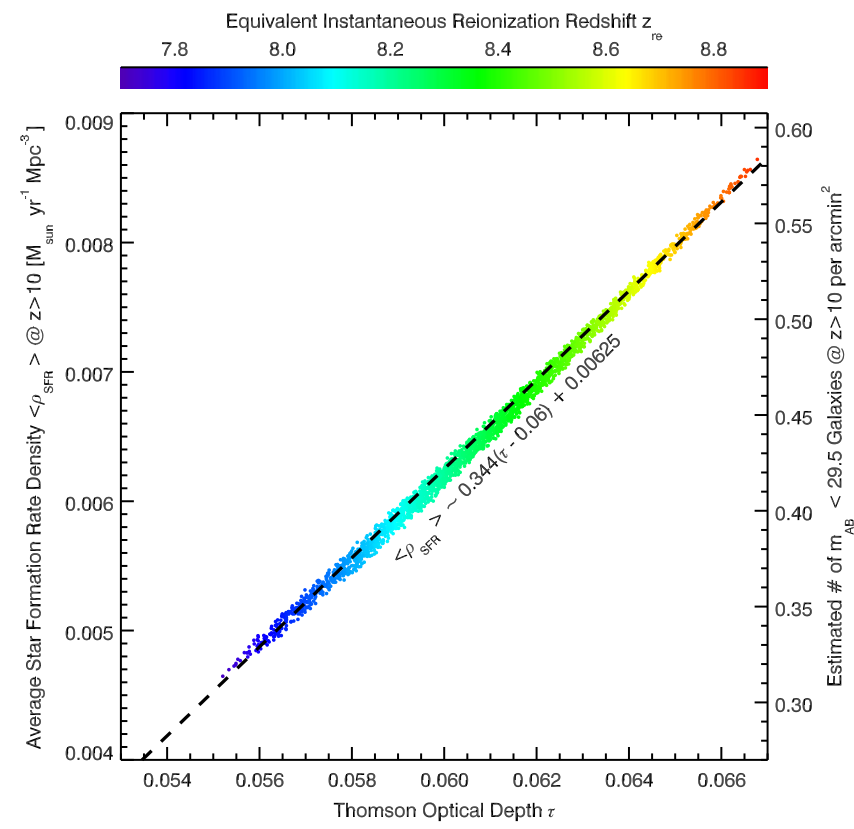

Figure 4. Correspondence between the Thomson optical depth, the equivalent instantaneous reionization redshift $z_{\text {reion }}$, and the average SFR density $\rho_{\mathrm{SFR}}$ at redshift $z \gtrsim 10$. Shown are samples (points) from the likelihood function of the $\rho_{\text {SFR }}$ model parameters resulting in the $68 \%$ credibility interval on $\tau$ from Figure 2, color coded by the value of $z_{\text {reion }}$. The samples follow a tight, nearly linear correlation (dashed line), demonstrating that in this model $\tau$ is a proxy for the high-redshift $\rho_{\mathrm{SFR}}$. We also indicate the number of $z>10$ galaxies with $m_{\mathrm{AB}}<29.5$ per $\operatorname{arcmin}^{-2}$ (right axis), assuming the LF shape does not evolve above $z>10$.

empirical limit on models that increase the ionizing efficiency during this epoch, e.g., with massive Population III stars and star formation in mini-halos (see Loeb \& Furlanetto 2013 for an overview of such models). Our results suggest such models cannot dramatically change the star formation efficiency at the earliest times.

Reionization proceeds relatively quickly as the ionized fraction evolves from $Q_{\mathrm{H}_{\text {II }}}=0.2$ to $Q_{\mathrm{H}_{\text {II }}}=0.9$ in only $400 \mathrm{Myr}$ of cosmic history over $6 \lesssim z \lesssim 9$. This duration is consistent with recent upper limits on the kinetic SunyaevZel'dovich effect (e.g., George et al. 2015). Our results offer extra hope for efforts to make redshifted $21 \mathrm{~cm}$ measurements of neutral hydrogen in the IGM, as the experimental foregrounds are weakest at low redshifts (e.g., Bowman et al. 2013; van Haarlem et al. 2013; Pober et al. 2014). Such experiments are essential for testing key assumptions in our analysis (such as $f_{\text {esc }}$ and $L_{\mathrm{min}}$ ) by observing the reionization process directly. The apparent lateness of reionization suggests that nextgeneration experiments, which hope to reach $z \sim 20$, can probe even earlier phases of galaxy formation.

We acknowledge useful discussions with George Efstathiou and Martin Haehnelt. This work was supported by Space Telescope Science Institute under award HST-GO-12498.01 A and the National Science Foundation under grant No. 1228509. J.S.D. acknowledges the support of the European Research
Council via the award of an Advanced Grant, and the contribution of the EC FP7 SPACE project ASTRODEEP (Ref. No. 312725).

\section{REFERENCES}

Atek, H., Kneib, J. P., Pacifici, C., et al. 2014, ApJ, submitted (arXiv:1409.0512)

Becker, G. D., \& Bolton, J. S. 2013, MNRAS, 436, 1023

Beckwith, S. V. W., Stiavelli, M., Koekemoer, A. M., et al. 2006, AJ, 132, 1729

Behroozi, P. S., \& Silk, J. 2015, ApJ, 799, 32

Bolton, J. S., Haehnelt, M. G., Warren, S. J., et al. 2011, MNRAS, 416, L70

Bouwens, R. J., Illingworth, G. D., Oesch, P. A., et al. 2014, ApJ, in press (arXiv:1403.4295)

Bouwens, R. J., Illingworth, G. D., Oesch, P. A., et al. 2012, ApJL, 752, L5

Bowman, J. D., Cairns, I., Kaplan, D. L., et al. 2013, PASA, 30, 31

Bromm, V., \& Yoshida, N. 2011, ARA\&A, 49, 373

Chornock, R., Berger, E., Fox, D. B., et al. 2013, ApJ, 774, 26

Choudhury, T. R., Puchwein, E., Haehnelt, M. G., \& Bolton, J. S. 2014, MNRAS, submitted (arXiv:1412.4790)

Dunlop, J. S. 2013, in Astrophysics and Space Science Library, ed. T. Wiklind, B. Mobasher, \& V. Bromm (Astrophysics and Space Science Library, Vol 396; Berlin: Springer), 223

Dunlop, J. S., Rogers, A. B., McLure, R. J., et al. 2013, MNRAS, 432, 3520

Fan, X., Strauss, M. A., Becker, R. H., et al. 2006, AJ, 132, 117

Feroz, F., Hobson, M. P., \& Bridges, M. 2009, MNRAS, 398, 1601

Finkelstein, S. L., Ryan, R. E., Jr., Papovich, C., et al. 2014, ApJ, submitted (arXiv:1410.5439)

George, E. M., Reichardt, C. L., Aird, K. A., et al. 2015, ApJ, 799, 177

Hinshaw, G., Larson, D., Komatsu, E., et al. 2013, ApJS, 208, 19

Illingworth, G. D., Magee, D., Oesch, P. A., et al. 2013, ApJS, 209, 6

Ishigaki, M., Kawamata, R., Ouchi, M., et al. 2015, ApJ, 799, 12

Koekemoer, A. M., Ellis, R. S., McLure, R. J., et al. 2013, ApJS, 209, 3

Kuhlen, M., \& Faucher-Giguère, C.-A. 2012, MNRAS, 423, 862

Loeb, A., \& Furlanetto, S. R. 2013, The First Galaxies in the Universe (Princeton, NJ: Princeton Univ. Press)

Madau, P., \& Dickinson, M. 2014, ARA\&A, 52, 415 (MD 14)

McGreer, I. D., Mesinger, A., \& D’Odorico, V. 2015, MNRAS, 447, 499

McLeod, D. J., McLure, R. J., Dunlop, J. S., et al. 2014, MNRAS, submitted (arXiv:1412.1472)

McLure, R. J., Dunlop, J. S., Bowler, R. A. A., et al. 2013, MNRAS, 432, 2696

Oesch, P. A., Bouwens, R. J., Illingworth, G. D., et al. 2014, ApJ, submitted (arXiv:1409.1228)

Oesch, P. A., Bouwens, R. J., Illingworth, G. D., et al. 2013, ApJ, 773, 75

Oesch, P. A., Bouwens, R. J., Illingworth, G. D., et al. 2012, ApJ, 745, 110

Oke, J. B. 1974, ApJS, 27, 21

Pawlik, A. H., Schaye, J., \& van Scherpenzeel, E. 2009, MNRAS, 394, 1812

Pentericci, L., Fontana, A., Vanzella, E., et al. 2011, ApJ, 743, 132

Pentericci, L., Vanzella, E., Fontana, A., et al. 2014, ApJ, 793, 113

Planck Collaboration, Ade, P. A. R., Aghanim, N., et al. 2015, arXiv: 1502.01589

Pober, J. C., Liu, A., Dillon, J. S., et al. 2014, ApJ, 782, 66

Robertson, B. E., Ellis, R. S., Dunlop, J. S., McLure, R. J., \& Stark, D. P. 2010, Natur, 468, 49

Robertson, B. E., Ellis, R. S., Dunlop, J. S., et al. 2014, ApJL, 796, L27

Robertson, B. E., Furlanetto, S. R., Schneider, E. E., et al. 2013, ApJ, 768, 71

Schenker, M. A., Ellis, R. S., Konidaris, N. P., \& Stark, D. P. 2014, ApJ, 795,20

Schenker, M. A., Robertson, B. E., Ellis, R. S., et al. 2013, ApJ, 768, 196

Schenker, M. A., Stark, D. P., Ellis, R. S., et al. 2012, ApJ, 744, 179

Shull, J. M., Harness, A., Trenti, M., \& Smith, B. D. 2012, ApJ, 747, 100

Stark, D. P., Ellis, R. S., Chiu, K., Ouchi, M., \& Bunker, A. 2010, MNRAS, 408, 1628

Tilvi, V., Papovich, C., Finkelstein, S. L., et al. 2014, ApJ, 794, 5

Topping, M. W., \& Shull, J. M. 2015, ApJ, 800, 97

Treu, T., Schmidt, K. B., Trenti, M., Bradley, L. D., \& Stiavelli, M. 2013, ApJL, 775, L29

van Haarlem, M. P., Wise, M. W., Gunst, A. W., et al. 2013, A\&A, 556, A2 